\title{
Regulation of the Risk of Exposure to Antibiotics in Milk
}

\section{Michel Bourin* and Florence Clénet}

University of Nantes, Nantes 44100, France

In the European Union, an effective and rigorous examination and registration system exists for placing medicinal products on the market. The registration process ensures that only products meeting defined standards of quality, safety and efficacy, which have been thoroughly tested and reviewed by independent experts, reach the market place. This procedure is ultimately designed to protect the public, animals and the environment from poor quality and unsafe medicines.

The process requires applicants to submit data supporting the safety, quality and efficacy of a product in a detailed dossier, which is reviewed by independent scientific committee working on behalf of the governmental agency. The scientific review examines all aspects of the product and includes a detailed risk-benefit analysis. The safety and efficacy of the product are monitored closely following its entry into the marketplace under statutory post-authorization surveillance or pharmacovigilance (PV) programs and the risk-benefit analysis is closely monitored and reassessed throughout the product's life.

In the veterinary field, the Marketing Authorization Holder (MAH) is required by Competent Authorities (CA) to actively collect, analyze and report any information on adverse events related to the use of a veterinary medicinal product (VMP) in accordance with the applicable legislation to the CA concerned.

Veterinary PV concerns monitoring, evaluating and improving the safety of VMPs with particular reference to adverse events in animals and human beings but also adverse events due to off-label use and investigation of the validity of the withdrawal period and of potential environmental problems.

The experience gained with the system for veterinary PV in the European Union (EU) has shown that investigation into reported adverse reactions to VMPs in order to identify potential problems is needed before a decision can be taken on whether or not regulatory action is necessary. The investigation can be triggered by events reported spontaneously, like the mandatory reports by the MAHs on serious adverse reactions in animals and all kinds of reactions in humans, or by the contents of periodic safety update reports which also contain non-serious adverse reactions.

Regulatory action means action for safety reasons taken by an EU CA that influences the status of a marketing authorization, ranging from modification of product literature including summary of the product characteristics or packaging update through the suspension or withdrawal.

In accordance with current legislation, the $\mathrm{MAH}$ is obliged to implement a Risk Management System whereby instructions are described to identify, assess, prevent or minimize risks relating to the use of a VMP based on information received trough PV.

Investigation may be initiated by the CA and/or the MAH for example when adverse reactions occur at incidences higher than a defined baseline or when a potential food safety problem is identified. In relation to $\mathrm{PV}$, food safety issues may for instance be related to extra-label use in respect to dose, route of administration, duration of treatment or use in a food producing species of a VMP containing substance for which no MRL has been established.

The MRL is the maximum concentration of residue accepted by the EU in a food product obtained from an animal that has received a
VMP or that has been exposed to a biocidal product for use in animal husbandry. The EU requires by law that foodstuff, such as meat, milk or eggs, obtained from such animals mustn't contain any residues that might represent a hazard to the health of the consumer.

Before a VMP intended for food-producing animals can be authorized in the EU, the safety of its pharmacologically active substances and their residues is first evaluated and these studies are included in the marketing authorization dossier.

The MRL is regulatory and applies to a substance for a specific foodstuff equally in the EU. Compliance with MRLs entails criminal liability of the producer of the food. Numerous official controls are carried in all EU states. Progress at the analytical level allows detecting the presence of residues in food at ever lower levels, even lower than MRLs.

The withdrawal period defined in the MA is the time between the last use of the product and the release for consumption of foodstuffs from treated animals. It is defined so that foodstuffs from treated animals don't contain any residues at levels above the MRL. Beyond the threshold of the MRL, the food is not allowed.

In 2011, the French Authority (ANSES) was consulted by the DGAL on the validation of methods for the detection of antibiotic residues in milk for human consumption (a rapid screening test and a confirmation method) implemented as part of self-control performed by interprofessionnal laboratories of milk analysis.

Tetracycline's, beta-lactam antibiotics and cephalosporin's are families of molecules which represent the highest risk in terms of public health and are the most commonly prescribed in dairy production.

It is necessary to improve the performance and validity of tests used in screening and confirmation to detect molecules families such as macrolides', quinolones' and aminoglycosides' also used in dairy cows. The first step was to identify the molecules responsible for the positive samples for qualitative rapid tests. Validation studies were conducted to a method for rapid screening and confirmation. These milk samples were analyzed by liquid chromatography coupled with mass spectrometry (LC-MS/MS) to identify the compounds present in order to compare the results of both tests.

The studies showed tetracycline's (tetracycline and oxytetracycline); beta-lactam antibiotics (cephalonium, amoxicillin, penicillin G, cephapirin, cloxacillin) and aminosids (dihydrostreptomycin, neomycin) are the most likely molecules to be present in cow's milk.

Among 120 positive samples for quality test, half were positive to confirmation test. $37.5 \%$ of them didn't contain detectable molecules

*Corresponding author: Michel Bourin, University of Nantes, Nantes 44100, France, E-mail: michel.bourin@univ-nantes.fr

Received March 04, 2014; Accepted March 05, 2014; Published March 19, 2014

Citation: Bourin M, Clénet F (2014) Regulation of the Risk of Exposure to Antibiotics in Milk. Pharmaceut Reg Affairs 3: e128. doi:10.4172/2167-7689.1000e128

Copyright: ( 2014 Bourin M, et al. This is an open-access article distributed under the terms of the Creative Commons Attribution License, which permits unrestricted use, distribution, and reproduction in any medium, provided the original author and source are credited. 
targeted by the LC-MS/MS method. The confirmation test used confirmed the presence of residues of beta-lactams and tetracycline with a response rate from $1 / 4$ to $1 / 2$ MRL observed in the study and a low rate of false positive molecules. This combination of tests therefore has a detection capability generally below the MRL for the two major classes of molecules with the highest probability of being present in the milk. Traces of other molecules were observed at levels below the MRL in a significant proportion of samples but positive screening test can't always be explained by these observed traces.

Traces of gamithromycin have been highlighted in several samples and this finding has been reported to the National Agency for Veterinary Medicinal Products (ANMV) as part of Pharmacovigilance. This molecule has no MRL for milk and its use is not allowed in lactating females. It can however be administered to pregnant females under condition of withdrawal period respect.

Thus, such a self-control strategy reduces the risk of consumption of milk in which the highest risk of exceeding MRLs for molecules most commonly used and the risk of occurrence of other important molecules to concentrations above the MRL is very low.

On the one hand, the risk of exposure to these antibiotics through food is extremely low for the consumer. On the other hand, the economic consequences for producers of the penalties did not impose after detection of residues in a tank containing several thousand liters of milk without identification nor dosage of the suspected molecule may be very detrimental. 